\title{
$D$ meson production asymmetry, unfavored fragmentation, and consequences for prompt atmospheric neutrino production
}

\author{
Rafał Maciuła $^{*}$ and Antoni Szczurek ${ }^{\dagger}$ \\ Institute of Nuclear Physics, Polish Academy of Sciences, Radzikowskiego 152, \\ PL-31-342 Kraków, Poland
}

(Received 10 January 2018; published 2 April 2018)

\begin{abstract}
We consider unfavored light quark/antiquark to $D$ meson fragmentation. We discuss nonperturbative effects for small transverse momenta. The asymmetry for $D^{+}$and $D^{-}$production measured by the LHCb collaboration provides natural constraints on the parton (quark/antiquark) fragmentation functions. We find that already a fraction of $q / \bar{q} \rightarrow D$ fragmentation probability is sufficient to account for the measured asymmetry. We make predictions for similar asymmetry for neutral $D$ mesons. Large $D$-meson production asymmetries are found for large $x_{F}$ which is related to dominance of light quark/antiquark $q / \bar{q} \rightarrow D$ fragmentation over the standard $c \rightarrow D$ fragmentation. As a consequence, prompt atmospheric neutrino flux at high neutrino energies can be much larger than for the conventional $c \rightarrow D$ fragmentation. The latter can constitute a sizeable background for the cosmic neutrinos claimed to be observed recently by the IceCube Observatory. Large rapidity-dependent $D^{+} / D^{-}$and $D^{0} / \bar{D}^{0}$ asymmetries are predicted for low $(\sqrt{s}=20-100 \mathrm{GeV})$ energies. The $q / \bar{q} \rightarrow D$ fragmentation leads to enhanced production of $D$ mesons at low energies. At $\sqrt{s}=20 \mathrm{GeV}$ the enhancement factor with respect to the conventional contribution is larger than a factor of five. In the considered picture the large- $x_{F} D$ mesons are produced dominantly via fragmentation of light quarks/antiquarks. Predictions for fixed target $p+{ }^{4} \mathrm{He}$ collisions relevant for a fixed target LHCb experiment are presented.
\end{abstract}

DOI: 10.1103/PhysRevD.97.074001

\section{INTRODUCTION}

It is believed that the $D$ mesons are produced dominantly via $c \rightarrow D$ fragmentation. However, asymmetries for $D^{+}$ and $D^{-}$production were obtained at lower energies for $\pi^{-}$nucleus collisions [1] and $\Sigma^{-}$-nucleus collisions [2] and recently at the LHC for proton-proton collisions [3]. Rather small asymmetries of the order of $1 \%$ were found by the LHCb collaboration [3]. One can believe in such low asymmetries as the $C P$ asymmetries in decay defined as:

$$
A_{C P}=\frac{\Gamma\left(D^{+}\right)-\Gamma\left(D^{-}\right)}{\Gamma\left(D^{+}\right)+\Gamma\left(D^{-}\right)}
$$

were found to be extremely small, consistent with zero (see, e.g., Refs. [4-6] and references therein). The LHCb result was obtained for $D^{ \pm} \rightarrow K_{s}^{0} K^{ \pm}$decays.

*rafal.maciula@ifj.edu.pl

†antoni.szczurek@ifj.edu.pl

Also at University of Rzeszów, PL-35-959 Rzeszów, Poland

Published by the American Physical Society under the terms of the Creative Commons Attribution 4.0 International license. Further distribution of this work must maintain attribution to the author(s) and the published article's title, journal citation, and DOI. Funded by SCOAP.
Can perturbative effects lead to any asymmetry? Higherorder pQCD and electroweak effects on $c \bar{c}$ asymmetry (both quark and antiquark registered) was studied in Ref. [7] for $E_{T}>20 \mathrm{GeV}$. The predicted effect was, however, rather small $(<1 \%)$, at least for the $\mathrm{LHCb}$ (pseudo)rapidity coverage $2<\eta<4$.

The intrinsic charm contribution [8] can also be important for forward charm production. Different models of intrinsic charm were proposed recently [9-11]. The simplest model includes only nonperturbative gluon splitting and gives equal amount of charm and anticharm in the initial states and cannot explain observed asymmetries in the production of $D$ and $\bar{D}$ mesons to be discussed in the present paper. The intrinsic charm was also discussed recently in the context of atmospheric neutrino production (see, e.g., Ref. [12]).

The production asymmetries were interpreted in Refs. $[13,14]$ as due to meson cloud mechanism and specific structure of the proton Fock components. The string model approach to the problem of heavy meson production and asymmetries in the production of heavy mesons was discussed in extent in Ref. [15]. The LHCb asymmetry was discussed also in the framework of heavy-quark recombination approach [16] (for earlier work see, e.g., Ref. [17]). Here there are four unknown parameters responsible for formation of $D$ mesons. It was shown that with some combination of parameters one can describe the LHCb data [16]. 

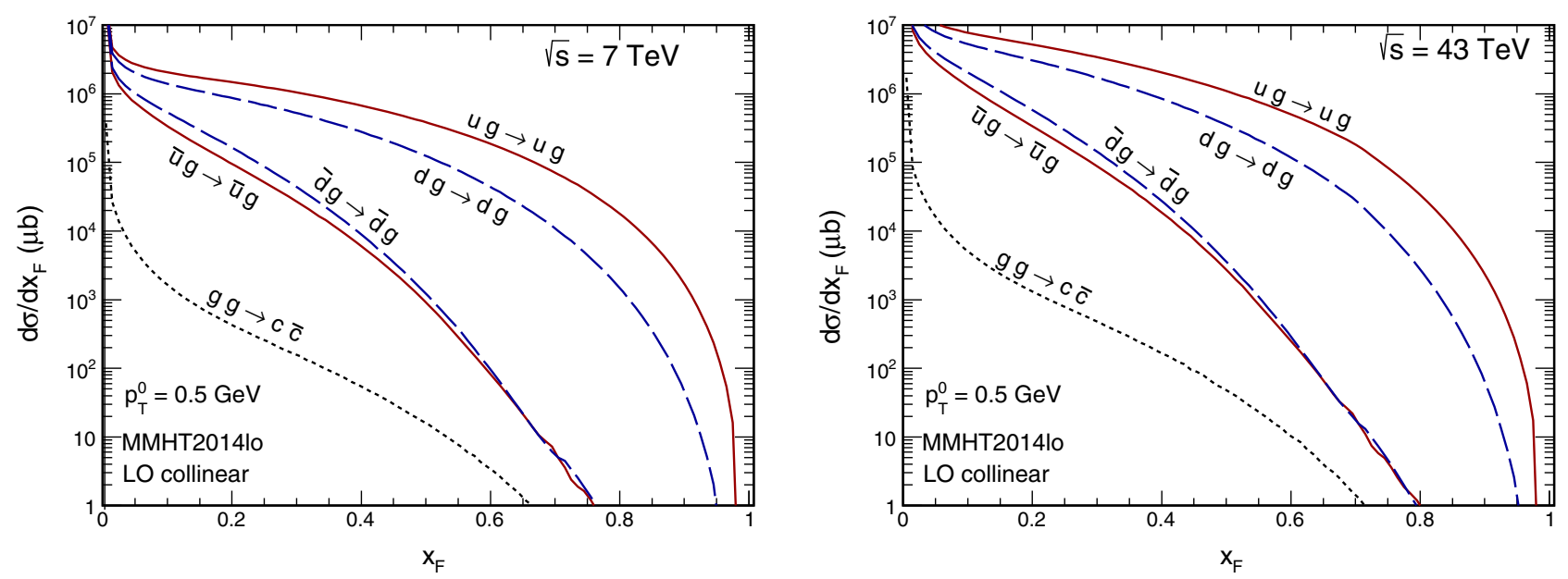

FIG. 1. Quark and antiquark distributions in Feynman $x_{F}$ for $\sqrt{s}=7 \mathrm{TeV}$ (left panel) and $\sqrt{s}=43 \mathrm{TeV}$ (right panel) corresponding to $E_{\text {lab }}(p)=10^{9} \mathrm{GeV}$ (relevant for high-energy prompt atmospheric neutrinos). This calculation was performed within collinearfactorization approach with $p_{T}^{0}=0.5 \mathrm{GeV}$.

The conventional $D$ meson production mechanism leads to symmetry in $D^{+} / D^{-}$or $D^{0} / \bar{D}^{0}$ production, i.e., $\sigma\left(D^{+}\right)=\sigma\left(D^{-}\right)$and $\sigma\left(D^{0}\right)=\sigma\left(\bar{D}^{0}\right)$. As will be discussed in the present paper, only a subtle isospin-violating effect in vector $D$ meson decays $\left(D^{*} \rightarrow D X\right)$ leads to a significant effect of $\sigma\left(D^{+} / D^{-}\right)<\sigma\left(D^{0} / \bar{D}^{0}\right)$.

Here we consider a simple alternative phenomenological explanation using so-called unfavored fragmentation functions responsible for light quark/antiquark fragmentation to $D$ mesons. Such unfavored fragmentation functions are known to be important, e.g., for $K^{+} / K^{-}$production and corresponding asymmetries obtained at SPS [18] and RHIC [19]. Such asymmetries for kaon production were nicely explained in the picture of subleading parton fragmentation at low energies [20]. The unfavored fragmentation functions $g \rightarrow D, q / \bar{q} \rightarrow D$ that fulfill Dokshitzer-Gribov-LipatovAltarelli-Parisi (DGLAP) equations were discussed, e.g., in Ref. [21]. Even assuming that at the initial scale the fragmentation functions vanish, they naturally appear at larger scales. The parameters of fragmentation functions were found in some fits to the $e^{+} e^{-}$data [21]. It is interesting whether the so-obtained unfavored fragmentation functions can describe the observed experimentally asymmetries in proton-proton collisions.

In the present paper we wish to constrain the strength of $q \rightarrow D(\bar{q} \rightarrow D)$ fragmentation functions using the recent LHCb data for $D^{+} / D^{-}$asymmetry. Then we shall discuss $q / \bar{q} \rightarrow D^{ \pm}$contribution to $d \sigma / d x_{F}$ distributions. Possible consequences for lower energies and/or for prompt atmospheric neutrino production will be discussed.

\section{A THEORETICAL BASIS OF THE PRESENT CALCULATIONS}

In this section we briefly review basic ingredients needed in the present analysis.

\section{A. Light quark/antiquark production}

We start with high collision energies. We calculate the dominant at large $x_{F}$ high-energy processes: $u g \rightarrow u g$, $d g \rightarrow d g, \bar{u} g \rightarrow \bar{u} g$ and $\bar{d} g \rightarrow \bar{d} g$ and subsequent light quark/antiquark to D meson fragmentation and/or decays. The calculations are done in the leading-order (LO) collinear factorization approach with a special treatment of minijets at low transverse momenta, as adopted in PYTHIA, by multiplying standard cross section by a somewhat arbitrary suppression factor [22]

$$
F_{\text {sup }}\left(p_{T}\right)=\frac{p_{T}^{4}}{\left(\left(p_{T}^{0}\right)^{2}+p_{T}^{2}\right)^{2}} \theta\left(p_{T}-p_{T, \text { cut }}\right) \text {. }
$$

First we calculate distributions of $u, d, \bar{u}, \bar{d}$ in Feynman $x_{F}$ in the forward (projectile) region. In Fig. 1 we show distributions in $x_{F}$ of the light-quarks/antiquarks obtained in the collinear-factorization approach. In this calculation we use the MMHT2014lo [23] parton distributions. The factorization and renormalization scales are taken as: $\mu_{F}^{2}$, $\mu_{R}^{2}=\mu_{0}^{2}+p_{T}^{2}$. Here we take $\mu_{0}^{2}=0.5^{2} \mathrm{GeV}^{2}$. In Fig. 2 we show results for different values of $p_{T}^{0}=0.5,1.0$, and $1.5 \mathrm{GeV}$. We think that already with $p_{T}^{0}=0.5 \mathrm{GeV}$ reliable quark/antiquark distributions in $y$ and $x_{F}$ are obtained. The shapes for different $p_{T}^{0}$ are rather similar.

At large $x_{F}$ the distribution of produced quarks/antiquarks can be approximated in terms of partons in the initial hadron as

$$
\frac{d \sigma}{d x_{F}}\left(x_{F}\right) \approx C(\sqrt{s}) x_{F} q_{f}\left(x_{F}, \mu_{\mathrm{eff}}^{2}\right),
$$

where $\mu_{\text {eff }}^{2}$ is the scale relevant for low transverse momentum quark/antiquark production. In Fig. 3 we compare results of calculations performed in the collinear-factorization 


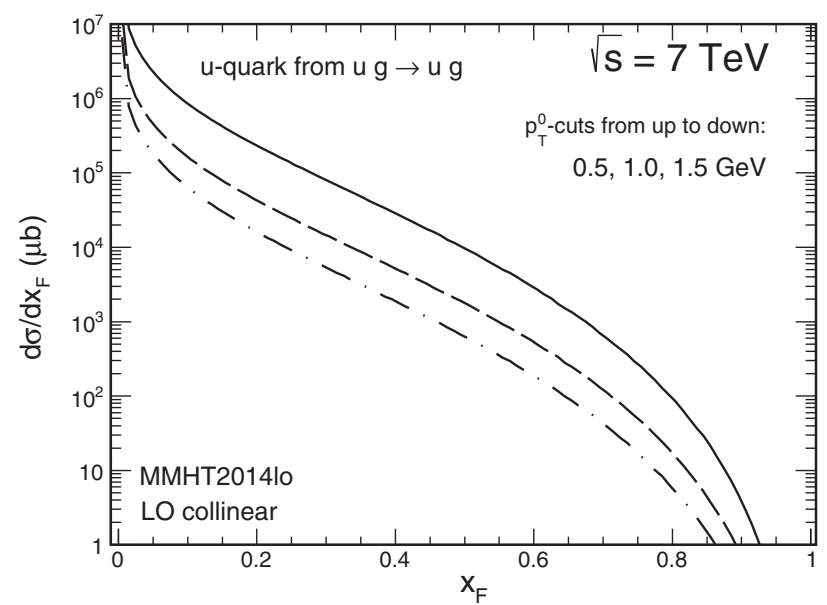

FIG. 2. Light u-quark distribution in Feynman $x_{F}$ for $\sqrt{s}=$ $7 \mathrm{TeV}$ for different values of $p_{T}^{0}=0.5,1.0$, and $1.5 \mathrm{GeV}$.

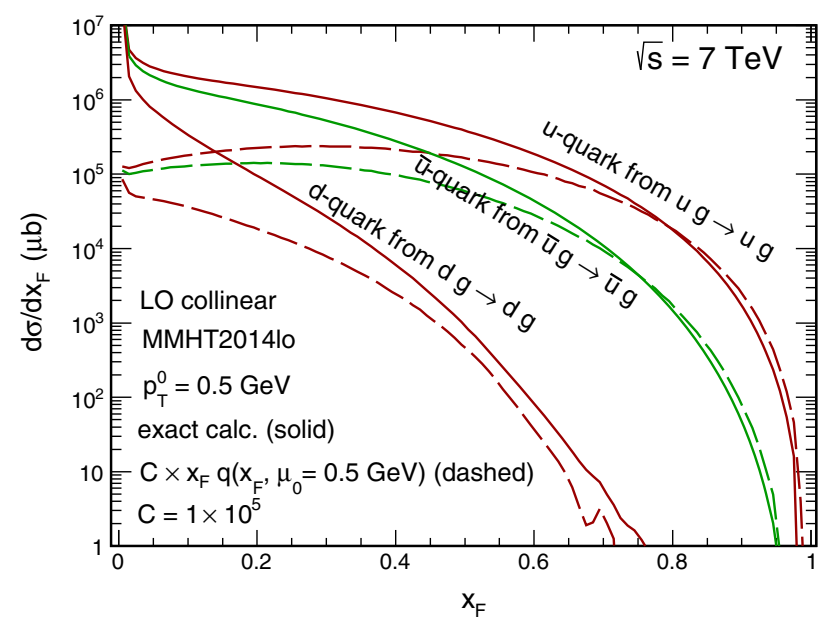

approach with those obtained with the very simple approximation given by Eq. (2.2). We see a reasonably good agreement of the results of the two calculations. The same parton distribution set was used in both cases. In this calculation $\mu_{\text {eff }}=0.5-3 \mathrm{GeV}$ was taken. The agreement for $u$ and $d$ quarks is much better than that for $\bar{u}$ and $\bar{d}$ antiquarks. The best agreement is obtained for $\mu_{\text {eff }} \approx 2-3 \mathrm{GeV}$.

The dependence on transverse momentum of quarks/ antiquarks is very steep. In Fig. 4 we show the transverse momentum distribution of produced light quarks and antiquarks. Here we have assumed a lower cut on $x_{F}>$ 0.2 to concentrate on the interesting for us region related to fast prompt atmospheric neutrinos [24]. Although there is a strong dependence of the cross section on $p_{T}$ the integrated cross sections are finite. The averaged transverse momentum is $p_{T} \sim 2 \mathrm{GeV}$.

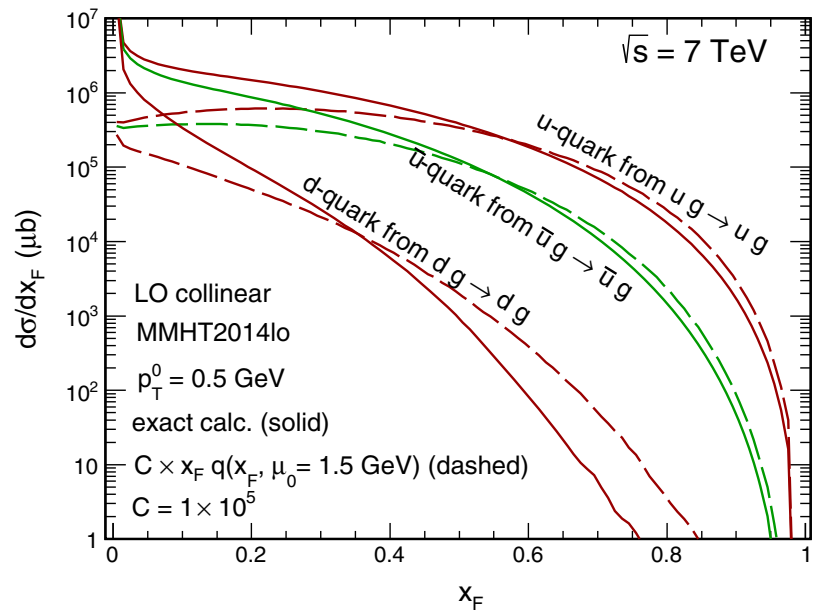

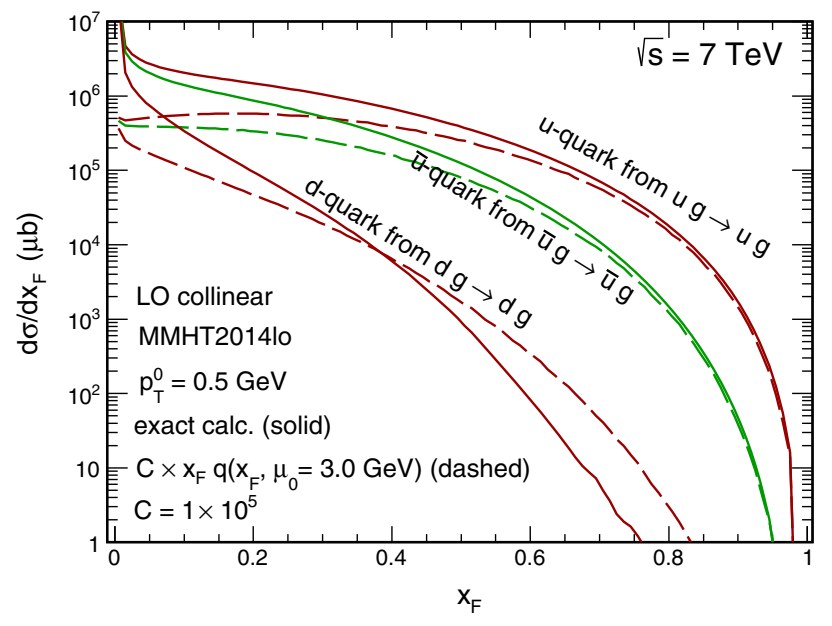

FIG. 3. Distribution in Feynman $x_{F}$ for $u$ and $d$ quarks and $\bar{u}$ antiquarks calculated with formula (2.2) for different factorization scales given explicitly in the figures. 


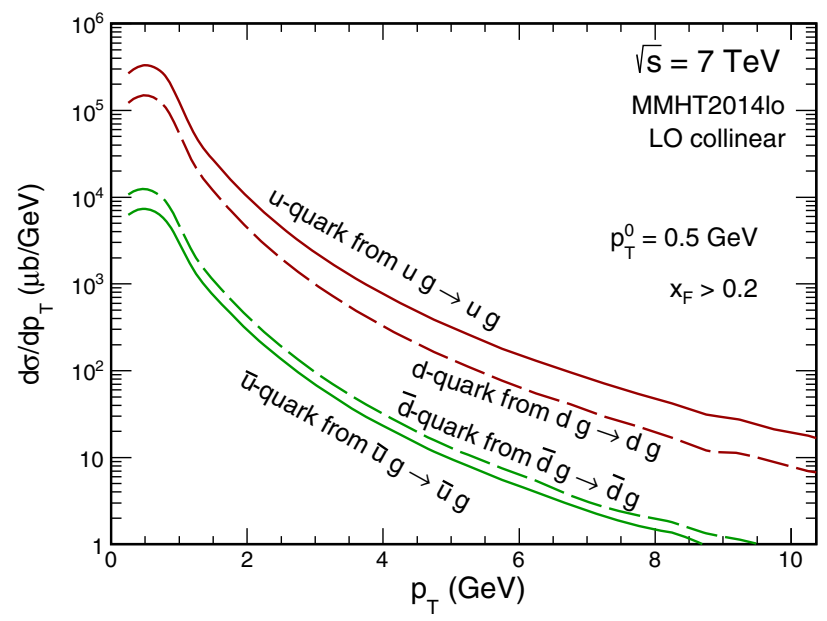

FIG. 4. Transverse momentum distribution of light quarks/ antiquarks for $x_{F}>0.2$.

\section{B. Unfavored fragmentation functions}

Let us start with direct fragmentation. Then we have to include $u, \bar{u}, d, \bar{d} \rightarrow D^{i}$ parton fragmentation. The corresponding fragmentation functions fulfill the following flavor symmetry conditions:

$$
D_{d \rightarrow D^{-}}(z)=D_{\bar{d} \rightarrow D^{+}}(z)=D^{(0)}(z) .
$$

Similar symmetry relations hold for fragmentation of $u$ and $\bar{u}$ to $D^{0}$ and $\bar{D}^{0}$ mesons. However $D_{q \rightarrow D^{0}}(z) \neq D_{q \rightarrow D^{+}}(z)$ which is caused by the contributions from decays of vector $D^{*}$ mesons. Furthermore we assume for doubly suppressed fragmentations:

$$
D_{\bar{u} \rightarrow D^{ \pm}}(z)=D_{u \rightarrow D^{ \pm}}(z)=0 .
$$

The fragmentation functions at sufficiently large scales undergo DGLAP evolution equations [21]. In the case of $e^{+} e^{-}$collisions the scale is usually taken as $\mu^{2}=s$. When fitting fragmentation functions to $e^{+} e^{-} \rightarrow D$ data one usually assumes

$$
D_{q / \bar{q} \rightarrow D}\left(z, \mu_{0}^{2}\right)=0
$$

at some initial scale usually taken as $\mu_{0}=m_{c}, 2 m_{c}$, where $m_{c}$ is charm quark mass. This simplification is not a good approximation for the case of proton-proton collisions where the asymmetry was observed [3] even at very low transverse momenta. Here we are particularly interested in low transverse momentum $D$ mesons. Then our typical factorization scales $\mu^{2}=p_{T}^{2}+m_{q}^{2}$ are very small. Therefore we limit in the following to a phenomenological approach and ignore possible DGLAP evolution effects important at somewhat larger transverse momenta. We can parametrize the unfavored fragmentation functions in this phase space region as:

$$
D_{q \rightarrow D}(z)=A_{\alpha}(1-z)^{\alpha} .
$$

Instead of fixing the unknown $A_{\alpha}$ we will operate rather with the fragmentation probability:

$$
P_{q \rightarrow D}=\int d z A_{\alpha}(1-z)^{\alpha} .
$$

and calculate corresponding $A_{\alpha}$ for a fixed $P_{q \rightarrow D}$ and $\alpha$. Therefore in our effective approach we have only two free parameters.

Another simple option one could consider is

$$
D_{q_{f} \rightarrow D}(z)=P_{q_{f} \rightarrow D} \cdot D_{\text {Peterson }}(1-z) .
$$

Then $P_{q_{f} \rightarrow D}$ would be the only free parameter. For heavy quark fragmentation $(c \rightarrow D)$ the Peterson fragmentation function is peaked at large $z$. However, the light quark/ antiquark fragmentation is expected to be dominant at small $z$. This is the case of Peterson fragmentation function reflected with respect to $z=1 / 2$. We use such a function purely phenomenologically to test uncertainties related to the shape of the a priori unknown function.

In addition to the direct fragmentation [given by $D^{(0)}(z)$ ] there are also contributions with intermediate vector $D^{*}$ mesons. Then the chain of production of charged $D$ mesons is naively as follows:

$$
\begin{aligned}
& \bar{u} \rightarrow D^{*, 0} \rightarrow D^{+} \quad \text { (forbidden) } \\
& u \rightarrow \bar{D}^{*, 0} \rightarrow D^{-} \quad \text { (forbidden) } \\
& \bar{d} \rightarrow D^{*,+} \rightarrow D^{+} \quad \text { (allowed) } \\
& d \rightarrow D^{*,-} \rightarrow D^{-} \quad \text { (allowed) }
\end{aligned}
$$

In reality the first two chains are not possible as the decays of corresponding vector mesons $\left(D^{*, 0}\right.$ and $\left.\bar{D}^{*, 0}\right)$ are forbidden by lack of phase space. This would be, however, possible for $D^{0}$ and $\bar{D}^{0}$ production where $D^{*, \pm}$ may decay producing $D^{0}$ or $\bar{D}^{0}$ mesons. In the latter case the two terms have different flavor structure and the production asymmetry is more complicated. In addition $D^{0}-\bar{D}^{0}$ oscillations occur (see, e.g., Refs. $[25,26]$ ) which makes the extraction of initial $D^{0} / \bar{D}^{0}$ production asymmetry a bit more difficult. According to our knowledge this has not been studied so far by the LHCb collaboration.

Including both direct and resonant contributions the combined fragmentation function of light quarks/antiquarks to charged $D$ mesons can be written as:

$D_{d / \bar{d} \rightarrow D^{\mp}}^{\text {eff }}(z)=D_{d / \bar{d} \rightarrow D^{\mp}}^{0}(z)+P_{\mp \rightarrow \mp} \cdot D_{d / \bar{d} \rightarrow D^{*, \mp}}^{1}(z)$.

The decay branching ratios can be found in Ref. [27] and is $P_{ \pm \rightarrow \pm}=0.323$. The indirect vector meson contributions 
have the same flavor structure as the direct one. It is easy to check that the decay $D^{*} \rightarrow D X$ practically does not change the distribution in $z$.

For neutral $D$ mesons we have similarly:

$$
\begin{array}{r}
D_{u / \bar{u} \rightarrow \bar{D}^{0} / D^{0}}^{\mathrm{eff}}(z)=D_{u / \bar{u} \rightarrow \bar{D}^{0} / D^{0}}^{0}(z)+P_{0 \rightarrow 0} \cdot D_{u / \bar{u} \rightarrow \bar{D}^{*, 0} / D^{*, 0}}^{1}(z), \\
D_{d / \bar{d} \rightarrow \bar{D}^{0} / D^{0}}^{\mathrm{eff}}(z)=P_{ \pm \rightarrow 0} \cdot D_{d / \bar{d} \rightarrow D^{*, \mp}}^{1}(z) .
\end{array}
$$

Here there are more possibilities than for charged $D$ mesons as both charged and neutral vector mesons decay into neutral $D$ mesons. The decay probabilities that appeared above are: $P_{0 \rightarrow 0}=0.667$ and $P_{ \pm \rightarrow 0}=1$ [27].

We assume flavor symmetry of fragmentation functions also for vector $D$ meson production:

$$
D_{u / \bar{u} \rightarrow \bar{D}^{* 0} / D^{*, 0}}^{1}(z)=D_{d / \bar{d} \rightarrow D^{*,}}^{1}(z)=D^{(1)}(z) .
$$

Finally we shall take an approximation:

$$
D^{(0)}(z) \approx D^{(1)}(z)
$$

which can be easily relaxed if needed. We think that such an approximation is, however, sufficient for the present exploratory calculations.

\section{C. $D$ meson distributions}

At forward directions (relevant for LHCb or IceCube) the details of hadronization are fairly important. Here the hadronization is done as in Ref. [20] assuming that the hadron pseudorapidity is equal to parton pseudorapidity and only momenta of hadrons are reduced compared to the parent partons.

In such an approximation the $D$ meson $x_{F}$-distributions at large $x_{F}$ can be obtained from the quark/antiquark distributions calculated in the collinear or $k_{t}$-factorization approaches as:

$$
\frac{d \sigma}{d x_{F}}=\sum_{f} \int_{x_{F}}^{1} \frac{d z}{z} \frac{d \sigma\left(x_{F} / z\right)}{d x_{F}^{\prime}} D_{q_{f} \rightarrow D}(z) .
$$

Instead of the more complicated calculations within collinear or $k_{t}$-factorization one can make first a simplified calculation. At very small transverse momenta and forward directions $\left(x_{F}>0.2\right)$ the outgoing quarks/antiquarks practically carry the same momentum fractions as the initial ones in the proton. Approximately one can therefore write the $x_{F}$ - distribution of outgoing quarks/antiquarks as

$$
\frac{d \sigma_{p p \rightarrow D X}\left(x_{F}\right)}{d x_{F}} \approx C \sum_{f} \int_{0}^{1} d z\left(x_{f} / z\right) q_{f}\left(x_{F} / z, \mu^{2}\right) D_{q_{f} \rightarrow D}(z) .
$$

The constant $C$ is responsible for the cross section normalization and depends on collision energy $C=C(\sqrt{s})$. The constant can be fitted to the asymmetries in experiments that measured different species of $D$ mesons.

\section{Flavor asymmetry}

Before we show our results for asymmetry we wish to show that the $k_{T}$-factorization approach with our choice of parameters gives a reasonable description of the data even at low transverse momenta. In Fig. 5 we show our results together with the LHCb data [28]. In this calculation we have taken $\operatorname{BR}\left(c \rightarrow D^{+}\right)=0.226$ (see Ref. [27]). The agreement is sufficiently good for our purpose even at small transverse momenta. It is not that good in the case of other approaches (see e.g., Refs. [29,30]).

Having shown that the $k_{T}$-factorization gives sufficiently good description of the experimental data we can study the $D^{+} / D^{-}$asymmetry.

The flavor asymmetry in production is defined as:

$$
A_{D^{+} / D^{-}}(\xi)=\frac{\frac{d \sigma_{D^{-}}}{d \xi}(\xi)-\frac{d \sigma_{D^{+}}}{d \xi}(\xi)}{\frac{d \sigma_{D^{-}}}{d \xi}(\xi)+\frac{d \sigma_{D^{-}}}{d \xi}(\xi)},
$$

where $\xi=x_{F}, y, p_{T},\left(y, p_{T}\right)$. In the following we shall consider several examples of selecting $\xi$.

To calculate asymmetry we have to include also dominant contribution corresponding to conventional $c / \bar{c} \rightarrow$ $D / \bar{D}$ fragmentation. The leading-order pQCD calculation is not reliable in this context. In the following the conventional contribution is calculated within the $k_{t}$-factorization approach with the Kimber-Martin-Ryskin unintegrated parton distributions [31] which has proven to well describe the LHC data. Such an approach seems consistent with

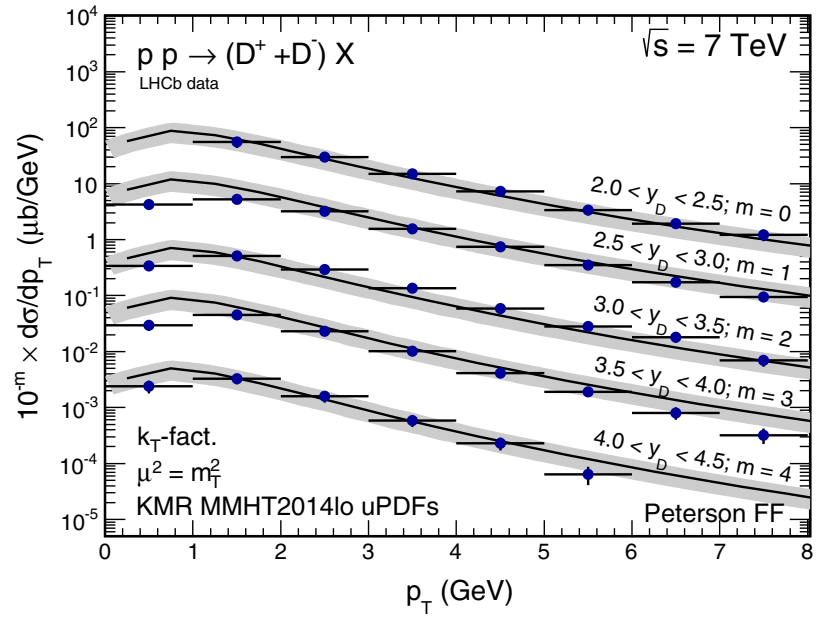

FIG. 5. Transverse momentum distributions of charged $D$ mesons for different windows of the $D$ meson rapidities specified in the figure. The experimental data points are from [28]. 

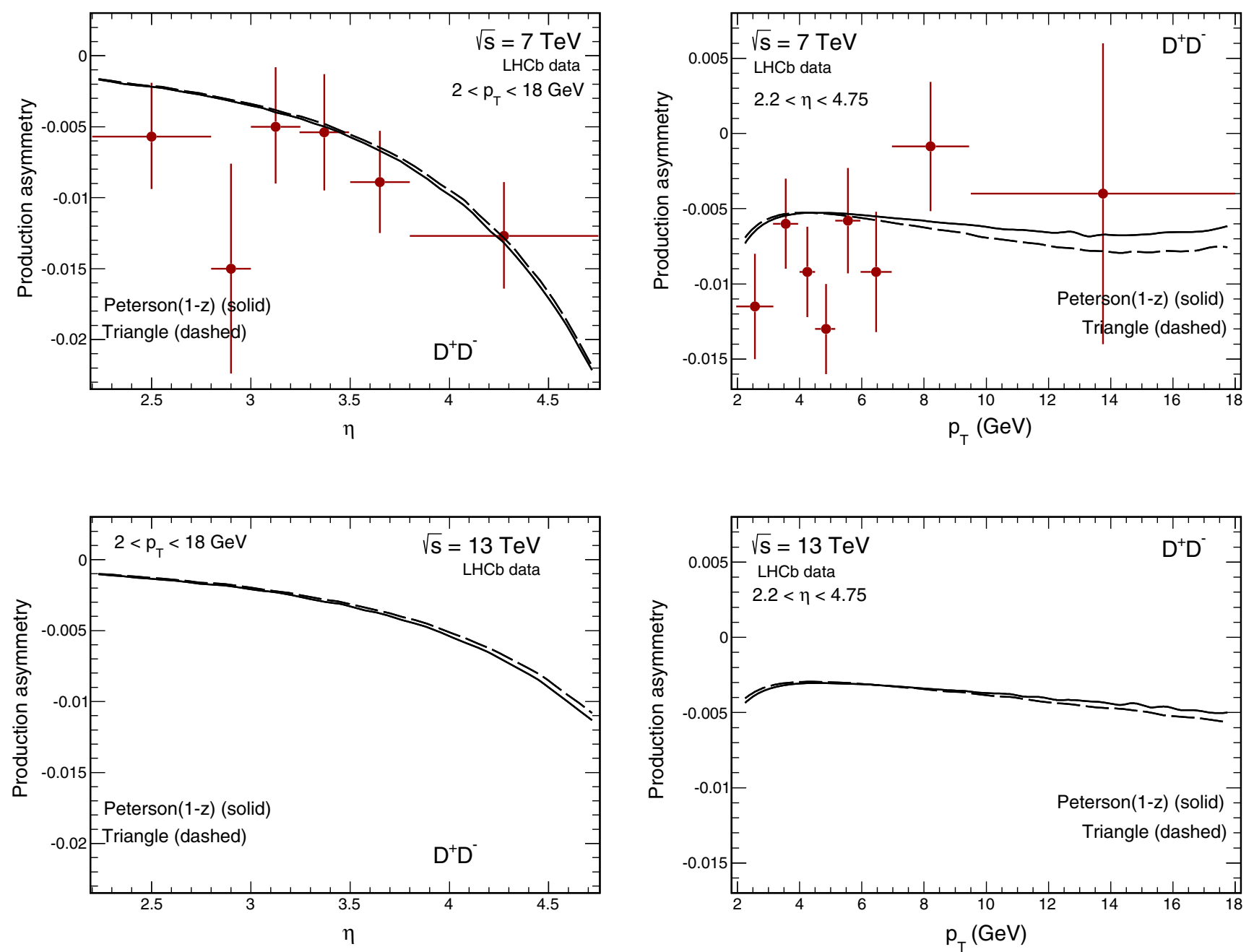

FIG. 6. $A_{D^{+} / D^{-}}$production asymmetry measured by the LHCb collaboration at $\sqrt{s}=7 \mathrm{TeV}$ as a function of $D$ meson pseudorapidity (left-top panel) and $D$ meson transverse momentum (right-top panel). The corresponding predictions for $\sqrt{s}=13 \mathrm{TeV}$ are shown in the bottom panels.

collinear next-to-leading order approach (see, e.g., a discussion in Ref. [32]).

For example in top panels of Fig. 6 we show results for the asymmetry for $P_{q \rightarrow D}$ adjusted to the LHCb data. In this calculation, and in the rest of the paper, we have fixed $\alpha=1$ in formula (2.6). We shall call corresponding fragmentation functions as triangular for brevity. In the left panel we show $A_{D^{+} / D^{-}}(\eta)$ for $p_{T, D} \in(2,18) \mathrm{GeV}$ and in the right panel we show $A_{D^{+} / D^{-}}\left(p_{T}\right)$ for $2.2<\eta<$ 4.75. We find that $P_{q \rightarrow D}=0.005 \pm 0.001$ for triangle fragmentation function and $P_{q \rightarrow D}=0.007 \pm 0.001$ for Peterson $(1-\mathrm{z})$ is consistent with main trends of the LHCb data. This are rather small numbers compared to $c / \bar{c} \rightarrow$ $D / \bar{D}$ fragmentation which happens with probability of the order of 50\%. The results do not depend on transverse momentum cut $p_{T}^{0}$, since the LHCb kinematics excludes the uncertain region of very small meson transverse momenta. In the bottom panels we show our predictions for $\sqrt{s}=13 \mathrm{TeV}$.
Charm conservation in strong processes must unavoidably lead to extra $c$ or $\bar{c}$ production at lower $x_{F}$ emitted rather in the remnant direction. The extra emissions lead to a reduction of asymmetries and enhanced production of charm (both mesons and baryons). This effect is not included explicitly when fitting the LHCb asymmetries. In our opinion the fit includes, however, this effect in an effective way.

Having described the $A_{D^{+} / D^{-}}$asymmetries for charged $D$ mesons we wish to make predictions for $A_{D^{0} / \bar{D}^{0}}$ production asymmetries for neutral $D$ mesons. According to our knowledge such asymmetries were never officially presented. The situation here is a bit more complicated due to $D^{0}-\bar{D}^{0}$ mixing and resulting oscillations. Here we calculate production asymmetry. In principle, the asymmetry may be (is) time dependent. However, the oscillation time seems much longer than the life time of $D^{0} / \bar{D}^{0}$ mesons, so it seems that the asymmetry could be measured 

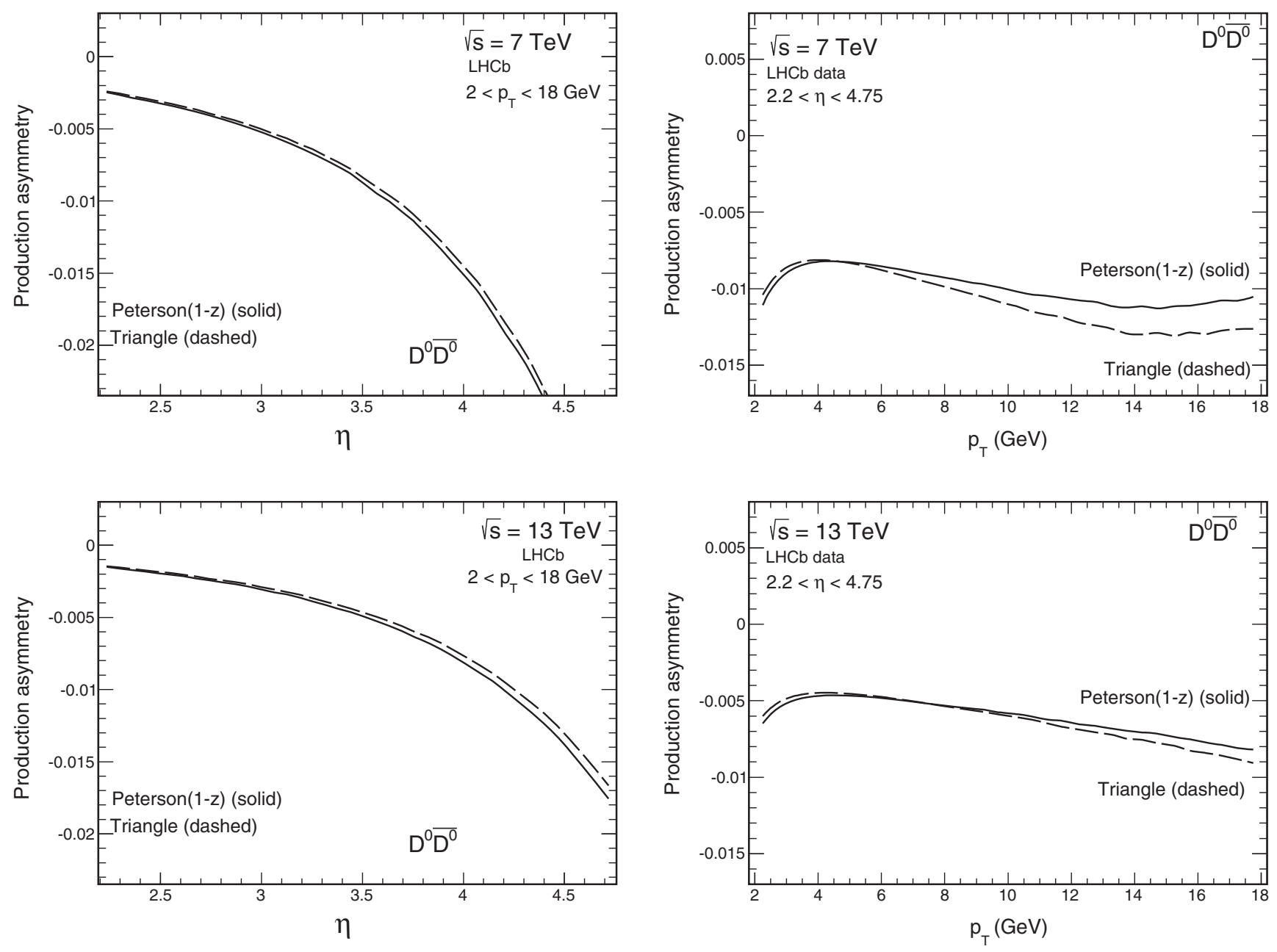

FIG. 7. $A_{D^{0} / \bar{D}^{0}}$ production asymmetry relevant for a possible LHCb collaboration measurement as a function of $D$ meson pseudorapidity (left panel) and $D$ meson transverse momentum (right panel). The corresponding predictions for $\sqrt{s}=7$ and 13 TeV are shown in the top and bottom panels, respectively.

experimentally, e.g., by the LHCb collaboration. This is very different for $B^{0} / \bar{B}^{0}$ mesons where the oscillation time is rather short. In Fig. 7 we show our predictions for asymmetries for neutral $D$ mesons. Slightly larger asymmetries are expected for $D^{0} / \bar{D}^{0}$ than for charged $D^{ \pm}$ mesons. $D^{0} / \bar{D}^{0}$ production symmetry is assumed in the $\mathrm{LHCb}$ studies of $C P$ violation [6]. Can such initial asymmetries have an influence on the extracted $A_{C P}$ for neutral $D$ mesons? This requires a separate dedicated study.

To supplement our results for asymmetry we wish to show how important is the subleading contribution for the LHC energy $\sqrt{s}=7 \mathrm{TeV}$. In Fig. 8 in addition to the conventional result (solid line) we show our estimate of the subleading fragmentation (dashed and dash-dotted lines). Clearly the subleading contribution is relatively small and starts to be sizeable only in the region of very small transverse momenta. For comparison we show also FONLL result [33] (dotted line). Here and in the following we use a default parameters set of the FONLL calculations, i.e.,

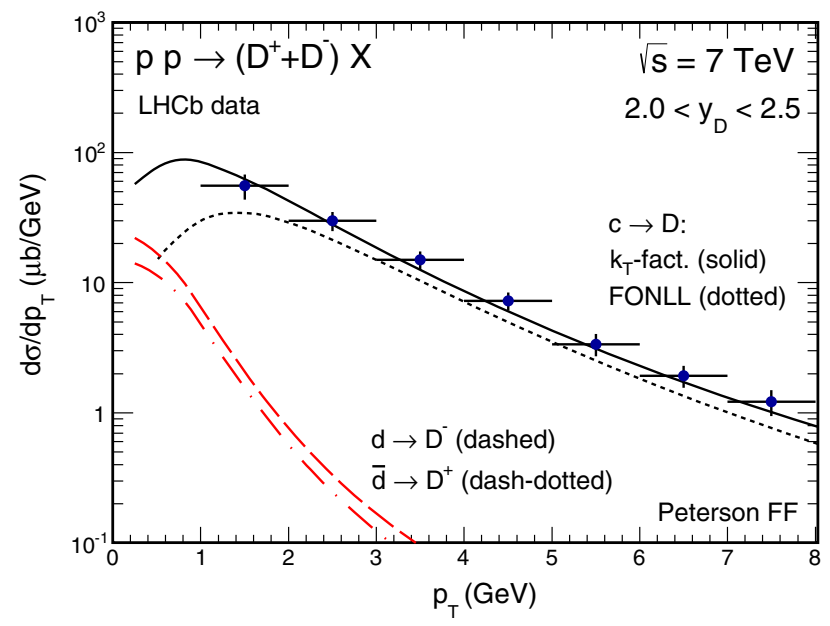

FIG. 8. Transverse momentum distributions of charged $D$ mesons from conventional (gluon-gluon fusion) and light quark/antiquark fragmentation for one selected window of the $D$ meson rapidities specified in the figure. The experimental data points are from [28]. 

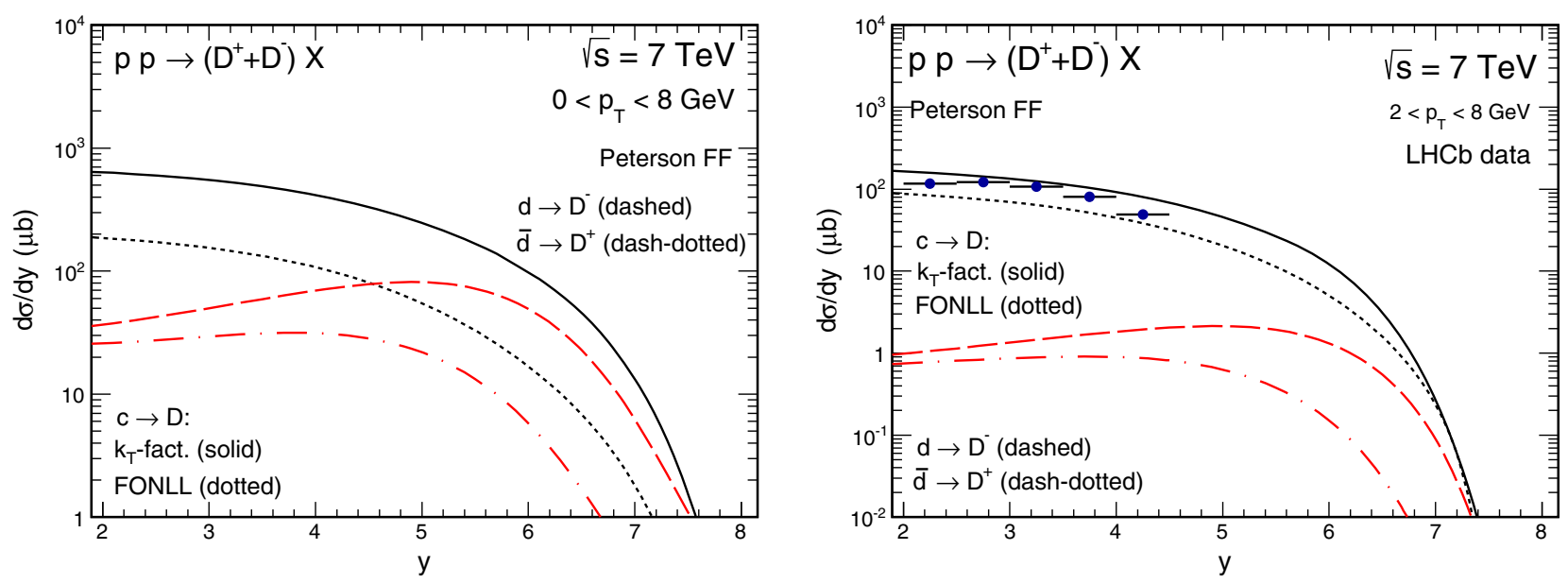

FIG. 9. Rapidity distribution of charged $D$ mesons from conventional (gluon-gluon fusion) and light quark/antiquark fragmentation integrated over $p_{t, D}$ in the range $0<p_{t, D}<8 \mathrm{GeV}$ (left panel) and $2<p_{t, D}<8 \mathrm{GeV}$ (right panel). The experimental data points were obtained by integrating $\left[(d \sigma) /\left(d p_{T}\right)\right]$ distributions from [28].

charm quark mass $m_{c}=1.5 \mathrm{GeV}$ and scales $\mu_{R}=\mu_{F}=\mu_{0}=$ $\sqrt{m_{c}^{2}+p_{T}^{2}}$ (the same as in the case of the $k_{T}$-factorization result).

For completeness in Fig. 9 we compare the two contributions as a function of $D$ meson rapidity. The subleading contributions start to be comparable to the conventional one only at large rapidities $y>5$, where no experimental coverage is available so far. For completeness we also show experimental results. The distributions were obtained by us via integrating $\frac{d \sigma}{d p_{T}} \mathrm{LHCb}$ distributions in different bins of rapidity. Here, our $k_{T}$-factorization result exceeds the experimental data if we go down to zero in transverse momentum, which is caused by the two lowest bins in $p_{T}$ (see left panel of Fig. 9). The description improves when extra condition $p_{T}>2 \mathrm{GeV}$ is imposed (see right panel of Fig. 9).

Now we shall make extrapolation to unmeasured regions. Assuming flavor symmetry for direct production of pseudoscalar and vector mesons [see Eq. (2.13)] we shall make predictions also for $D^{0}$ and $\bar{D}^{0}$ production.

\section{E. $D \bar{D}$ asymmetry at lower energies}

The asymmetry in $D^{+} / D^{-}$or $D^{0} / \bar{D}^{0}$ production is caused by the relative amount of $q / \bar{q} \rightarrow D$ and $c / \bar{c} \rightarrow$ $D$ fragmentation mechanisms. Here we include all partonic processes with light quark/antiquark in the final state. In Fig. 10 we show the asymmetries for three different energies $\sqrt{s}=20,50,100 \mathrm{GeV}$. We observe that the asymmetry at the lower energies is much larger than that for the LHC energies. Even at midrapidity $y \approx 0$ we predict sizeable asymmetries. Our rough predictions could be checked experimentally at SPS [18,34], RHIC or at fixed target $\mathrm{LHCb}$ [35]. Such experiments would allow to better pin down the rather weakly constrained so far $q / \bar{q} \rightarrow D$ fragmentation functions. Once this is done, a more realistic calculation for production of prompt neutrinos in the atmosphere could be done.

The discussed by us mechanisms of subleading fragmentation of $D$ mesons lead to enhanced production of $D$ mesons at lower energies. In Table I we show as an example different contributions to the production of $D^{+} / D^{-}$mesons. The dominant at high-energy $g g \rightarrow c \bar{c}$ mechanism gives only $13 \%$ and $18 \%$ for $\sqrt{s}=27$ and $39 \mathrm{GeV}$, respectively and strongly underestimates the NA27 [36] and E743 [37] experimental data. Inclusion of the "subleading" contributions brings theoretical calculations much closer to the experimental data. We predict sizeable $D^{+} / D^{-}$ asymmetries at these low energies, see Fig. 10.

The results shown in Table I require a bit more discussion. For instance it is known that the $k_{T}$-factorization approach gives considerably smaller cross section than next-to-leading collinear approach (see, e.g., Ref. [38]), where rather a hybrid model was used).

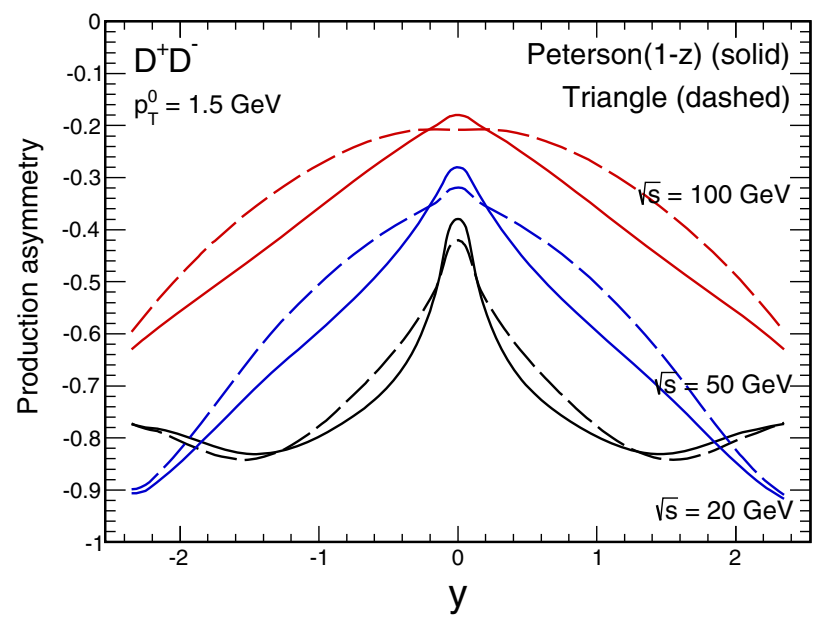

FIG. 10. $A_{D^{+} D^{-}}(y)$ production asymmetry in proton-proton collisions for different $\sqrt{s}$ indicated in the figure. 
TABLE I. Different contributions to the cross sections (in microbarns) for $D^{+}+D^{-}$production at low energies. The results presented here have been obtained with $p_{T}^{0}=1.5 \mathrm{GeV}$. The numbers from the two first rows are calculated in the $k_{T}$-factorization approach with off-shell initial state partons (highlighted by a star symbol). The rest of the results correspond to the LO collinear approximation.

\begin{tabular}{lcc}
\hline \hline Process: & $\sqrt{s}=27 \mathrm{GeV}$ & $\sqrt{s}=39 \mathrm{GeV}$ \\
\hline$g^{*} g^{*} \rightarrow c \bar{c}\left(c / \bar{c} \rightarrow D^{ \pm}\right)$ & 1.52 & 4.58 \\
$q^{*} \bar{q}^{*} \rightarrow c \bar{c}\left(c / \bar{c} \rightarrow D^{ \pm}\right)$ & 0.08 & 0.19 \\
$g d \rightarrow g d\left(d \rightarrow D^{-}\right)$ & 9.53 & 13.89 \\
$g \bar{d} \rightarrow g \bar{d}\left(\bar{d} \rightarrow D^{+}\right)$ & 3.03 & 4.78 \\
$d d \rightarrow d d\left(d \rightarrow D^{-}\right) \times 2$ & 3.07 & 4.29 \\
$\bar{d} \bar{d} \rightarrow \bar{d} \bar{d}\left(\bar{d} \rightarrow D^{+}\right) \times 2$ & 0.29 & 0.49 \\
$\bar{d} d \rightarrow \bar{d} d\left(d \rightarrow D^{-}\right)$ & 0.58 & 0.88 \\
$d \bar{d} \rightarrow d \bar{d}\left(\bar{d} \rightarrow D^{+}\right)$ & 0.58 & 0.88 \\
$u d \rightarrow u d\left(d \rightarrow D^{-}\right)$ & 2.76 & 3.72 \\
$\bar{u} \bar{d} \rightarrow \bar{u} \bar{d}\left(\bar{d} \rightarrow D^{+}\right)$ & 0.12 & 0.19 \\
$\bar{u} d \rightarrow \bar{u} d\left(d \rightarrow D^{-}\right)$ & 0.40 & 0.63 \\
$u \bar{d} \rightarrow u \bar{d}\left(\bar{d} \rightarrow D^{+}\right)$ & 0.97 & 1.42 \\
Theory predictions & 22.93 & 35.94 \\
Experiment & NA27: & E743: \\
& $11.9 \pm 1.5$ & $26 \pm 4 \pm 25 \%$ \\
\hline \hline
\end{tabular}

In Fig. 11 we show the lowest energy data for charged $D$ mesons in proton-proton collisions [36,37]. We show results of calculation in the $k_{T}$-factorization as well as results obtained with the code FONLL. Both the $k_{T}$-factorization as well as FONLL results are below experimental data extrapolated to the full phase space. Including also theoretical uncertainties, this leaves room for our subleading fragmentation contribution. In the present paper it was

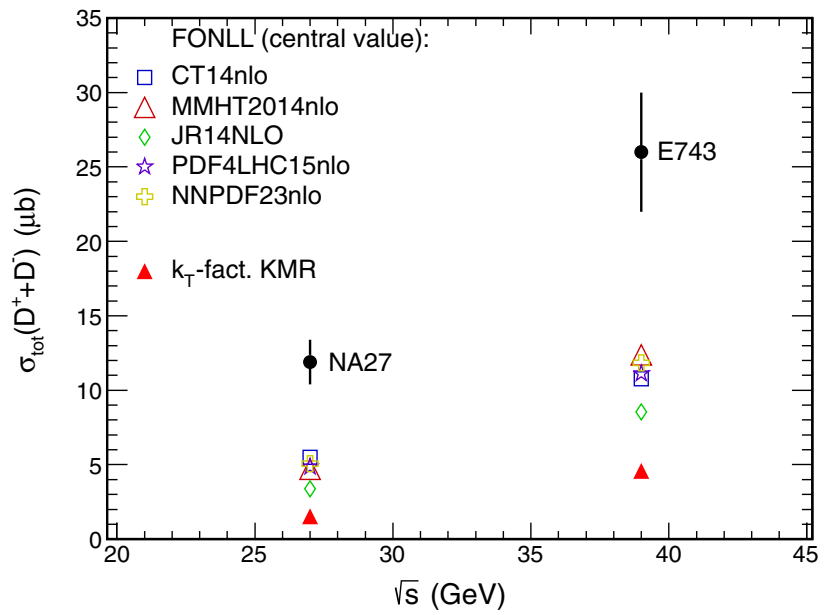

FIG. 11. Total cross section for $D^{+}+D^{-}$production. The experimental data are from Refs. [36,37]. The details of different calculations are explained in the figure. obtained by extrapolating our results, assuming some parametrizations of the subleading fragmentation function, to low energies based on the asymmetry measured by the $\mathrm{LHCb}$ collaboration. Of course our estimate to the $\mathrm{LHCb}$ asymmetry as well as extrapolation cannot be too precise. Clearly better data for intermediate and low energies are needed to constrain the subleading fragmentation. As discussed in the present paper some data may be expected relatively soon.

The LHCb collaboration has an experience in measuring the asymmetry in $D^{+}$and $D^{-}$production. It would be valuable to repeat such an analysis for fixed target experiment $p+{ }^{4} \mathrm{He}$ with gaseous target. The data have been already collected. The nuclear effects for ${ }^{4} \mathrm{He}$ should not be too large. Then the collision may be treated as a superposition of $p p$ and $p n$ collisions. Neglecting the nuclear effects the differential cross section (in the collinear factorization approach) for production of $q / \bar{q}$ (particle 1) and associated parton (particle 2) can be written approximately as:

$$
\frac{d \sigma_{p}{ }^{4} \mathrm{He}}{d y_{1} d y_{2} d p_{T}}=2 \frac{d \sigma_{p p}}{d y_{1} d y_{2} d p_{T}}+2 \frac{d \sigma_{p n}}{d y_{1} d y_{2} d p_{T}} .
$$

In the case of the second term we have to take into account parton (quark/antiquark) distribution in neutron which can be obtained from those in proton by assuming isospin symmetry between parton distributions in the proton and neutron. We are not interested in the distribution of gluons, that are treated here as inactive in the production of $D$ mesons. ${ }^{1}$ Therefore an integration over gluon variables is performed as previously.

In Fig. 12 we present the relevant predictions for the $\mathrm{LHCb}$ experiment. Rather large asymmetries are predicted which could be addressed in the forthcoming analysis of the fixed target experiment.

\section{F. Charge-to-neutral $D$ meson ratio}

In the standard pQCD approach (production of $c / \bar{c}$ and only $c / \bar{c} \rightarrow D / \bar{D}$ fragmentation) the ratio defined as

$$
R_{c / n} \equiv \frac{D^{+}+D^{-}}{D^{0}+\bar{D}^{0}}
$$

is a constant, independent of collision energy and rapidity (or $x_{F}$ ). Inclusion of the subleading contribution changes the situation. In Fig. 13 we show as an example the ratio as a function of meson pseudorapidity $\eta$ for LHC energies (left panel) and meson rapidity $y$ for $\sqrt{s}=100 \mathrm{GeV}$ (right panel), taking into account the subleading contribution.

\footnotetext{
${ }^{1}$ A possible active role of gluons was discussed, e.g., in Ref. [39] in the context of double parton scattering. Inclusion of the gluon fragmentation leads to much larger $\sigma_{\text {eff }}$, a parameter in the description of double-parton scattering.
} 


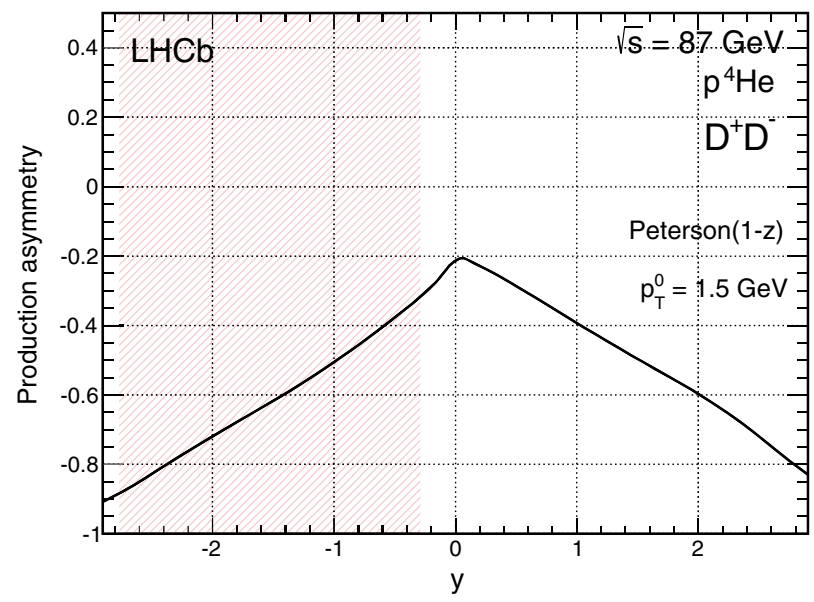

FIG. 12. $A_{D^{+} D^{-}}(y)$ production asymmetry for the fixed target $p+{ }^{4} \mathrm{He}$ reaction for $\sqrt{s}=87 \mathrm{GeV}$.

At the LHC energies very small, difficult to measure, effect is found for the LHCb transverse momentum and pseudorapidity range. At $\sqrt{s}=100 \mathrm{GeV}$ we predict a strong rapidity dependence of the $R_{c / n}$ ratio. Perhaps fixed target experiments at the LHCb could address the issue.

Identification of the dependence of $R_{c / n}$ on collision energy, rapidity or $x_{F}$ of $D$ mesons would be a good test of the considered here modeling and could better pin down the subleading fragmentation function.

\section{G. Resulting $D$ meson distributions and possible consequences for prompt neutrino flux}

In this subsection we wish to show results relevant for high-energy prompt atmospheric neutrinos. As discussed recently in Ref. [24] a rather large $x_{F} \sim 0.5$ region is important in this context. The $d \sigma / d x_{F}$ distribution of mesons is the most appropriate distribution in this context. For $x_{F}>0.1$ one can safely use the convolution formula from Eq. (2.15).

In Fig. 14 we compare the two contributions: (a) conventional one corresponding to $c \rightarrow D$ fragmentation and (b) subleading one corresponding to $q \rightarrow D$ fragmentation, for the sum of $D^{+}+D^{-}$(left panels) and $D^{0}+\overline{D^{0}}$ (right panels) mesons. While at small $x_{F}$ the conventional contribution dominates, at large $x_{F}$ the situation reverses. In addition we show the uncertainties bands where the upper and lower limits correspond to the predictions for $p_{T}^{0}=0.5$ and $1.5 \mathrm{GeV}$, respectively. The situation for both, $\sqrt{s}=7$ (top panels) and $43 \mathrm{TeV}$ (bottom panels), energies is rather similar. The enhancement due to the subleading contributions for neutral $D$ meson seems bigger than that for charged $D$ mesons (see Fig. 15). For example, for the triangle fragmentation functions, at $\sqrt{s}=43 \mathrm{TeV}$ for $x_{F} \sim 0.5$ the cross section for charged mesons $\left(D^{+}+D^{-}\right)$is $3-15$ times bigger than for conventional approach while the cross section for neutral mesons $\left(D^{0}+\bar{D}^{0}\right)$ is $20-200$ times bigger.

We predict also asymmetry for $D^{+} / D^{-}$and $D^{0} / \bar{D}^{0}$ production in the region of large $x_{F}$, relevant for IceCube. In Fig. 16 we show the asymmetry for the two large collision energies. Within our model we predict larger asymmetries at larger energy in this kinematical domain. Such asymmetries would lead to asymmetry in the production of neutrinos and antineutrinos. We do not know whether this could or not be measured.

The above results may have important consequences for large-energy atmospheric production which is not yet well understood background for cosmic (extraterrestrial) neutrinos, claimed to be observed by the IceCube collaboration [40]. This will be a topic of a forthcoming analysis.
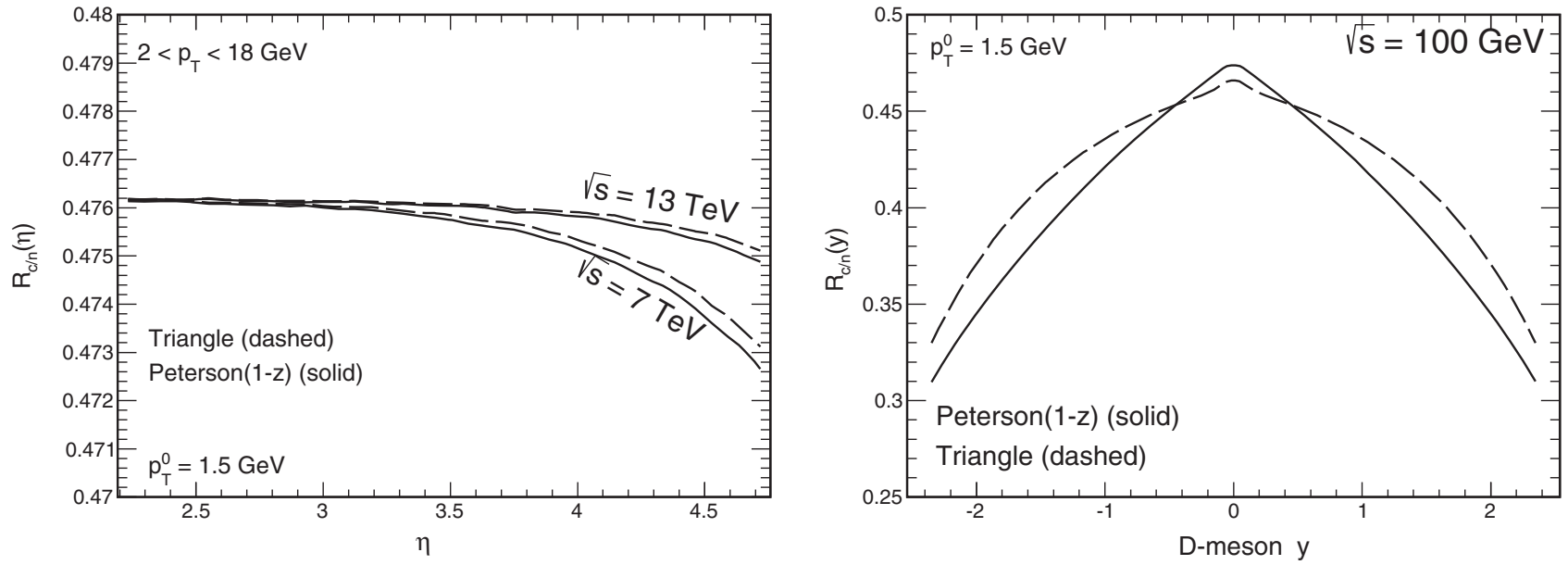

FIG. 13. The $R_{c / n}$ ratio as a function of meson pseudorapidity for $\sqrt{s}=7$ and $13 \mathrm{TeV}$ for the LHCb kinematics (left panel) and as a function of meson rapidity for $\sqrt{s}=100 \mathrm{GeV}$ in the full phase-space (right panel). Only quark-gluon subleading components are included here. 

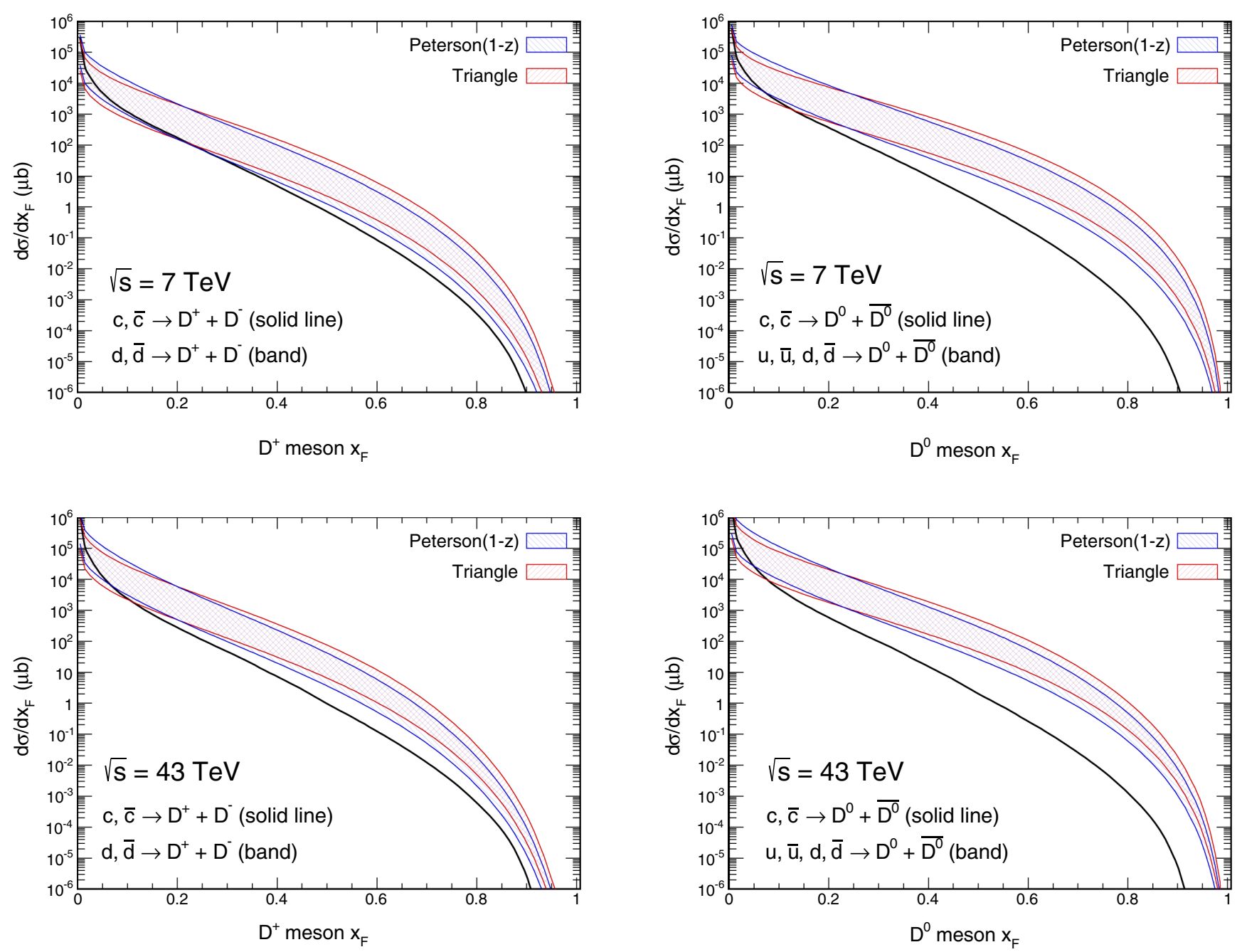

FIG. 14. Distribution in $x_{F}$ for charged $D^{+}+D^{-}$(left panel) and neutral $D^{0}+\bar{D}^{0}$ (right panel) $D$ mesons from conventional (solid lines) and subleading (shaded bands) mechanisms. The top panels are for $\sqrt{s}=7 \mathrm{TeV}$ and the bottom panels are for $\sqrt{s}=43 \mathrm{TeV}$.
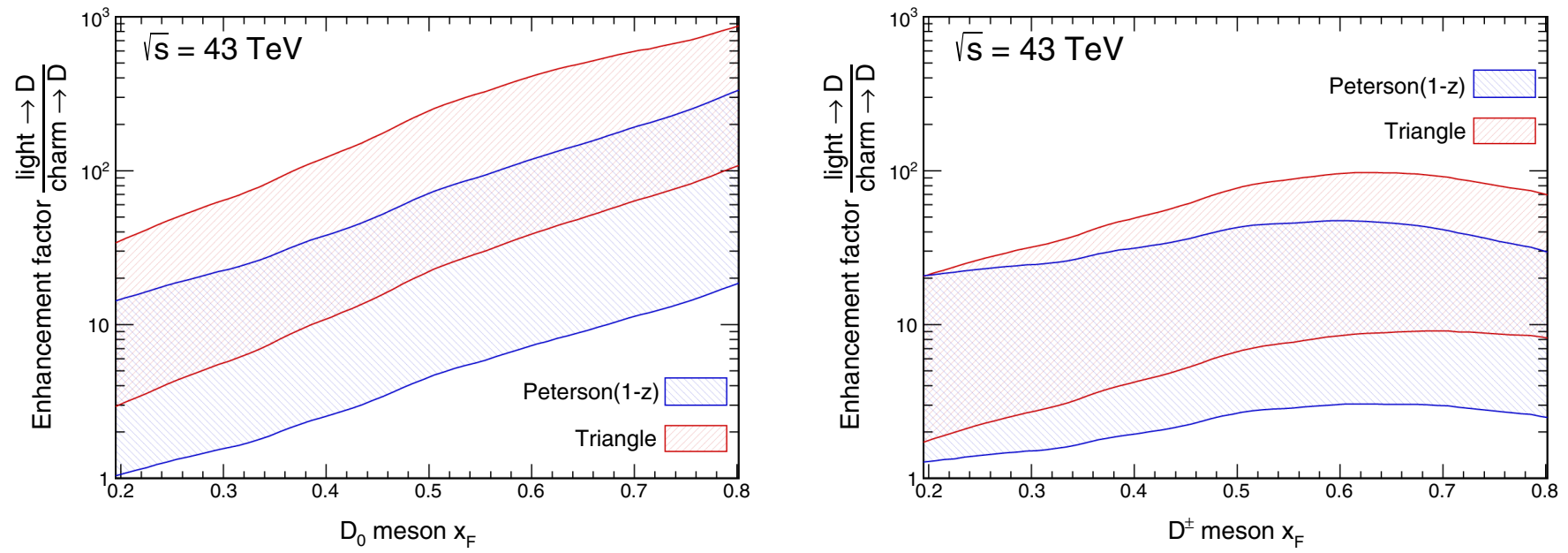

FIG. 15. Enhancement factor for neutral (left panel) and charged (right panel) charm meson for $\sqrt{s}=43 \mathrm{TeV}$. 

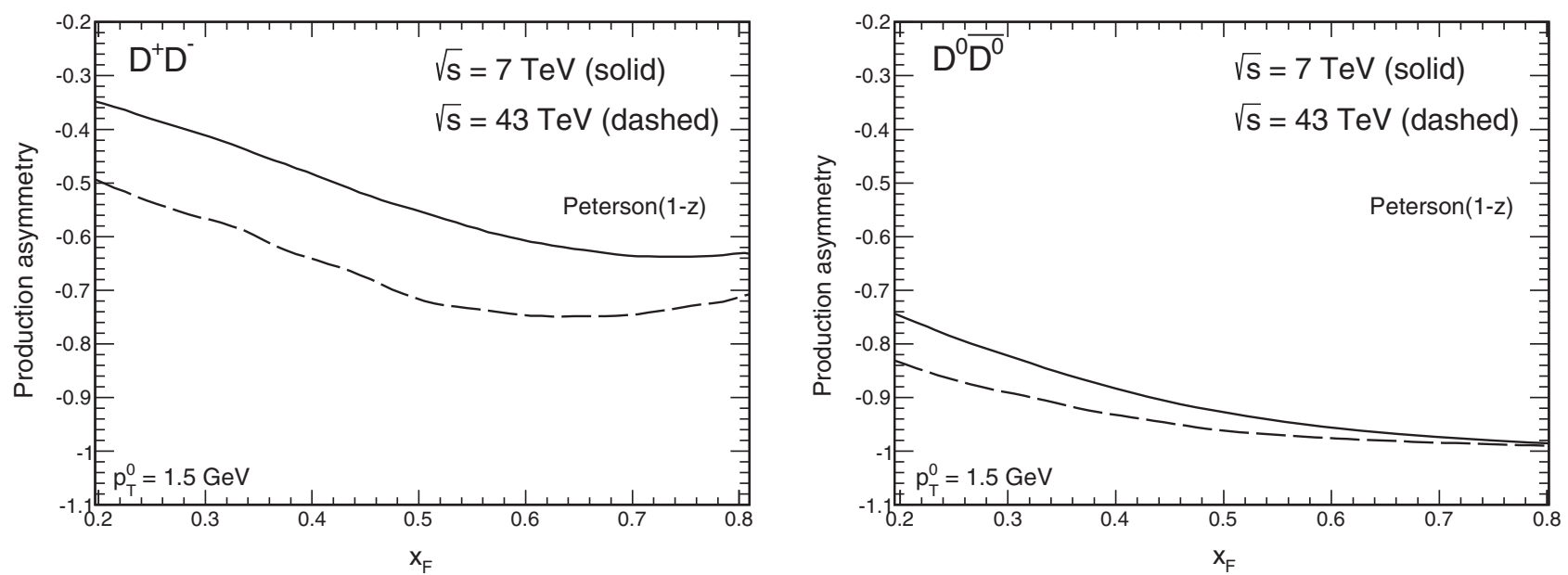

FIG. 16. Production asymmetry as a function of $x_{F}$ for $D^{+} / D^{-}$(left panel) and for $D^{0} / \overline{D^{0}}$ (right panel). The solid lines correspond to $\sqrt{s}=7 \mathrm{TeV}$ and the dashed lines correspond to $\sqrt{s}=43 \mathrm{TeV}$. The results are obtained with $p_{T}^{0}=1.5 \mathrm{GeV}$.

\section{CONCLUSIONS}

In the present paper we have discussed asymmetry in production of $D^{+}$and $D^{-}$mesons in proton-proton collisions. For a first time we have tried to understand whether the asymmetry observed by the LHCb collaboration can be understood within parton fragmentation picture, including light quark and antiquark fragmentation functions.

We have shown that our results for charged meson production nicely describe the forward $\mathrm{LHCb}$ data for all regions of rapidity and can be used as a reference to calculate the charged-meson asymmetries.

The light quark/antiquark fragmentation to $D$ mesons arises naturally within DGLAP evolution of fragmentation functions even assuming vanishing fragmentation functions at some initial scale. To understand the LHCb asymmetry we need, however, nonvanishing initial (for evolution) fragmentation functions. Very small initial unfavored fragmentation functions are sufficient to describe the $\mathrm{LHCb}$ data. The details depend on functional form used. The corresponding fragmentation probability for $q / \bar{q} \rightarrow D$ is very small, of the order of a fraction of $1 \%$, compared to $50 \%$ for $c / \bar{c} \rightarrow D$ fragmentation.

Having described the asymmetry for charged $D$ mesons we have made predictions for similar asymmetry for neutral $D$ mesons. Nonzero asymmetries have been predicted. This asymmetry may be, however, a bit more difficult to measure due to $D^{0}-\bar{D}^{0}$ oscillations confirmed recently experimentally.

Furthermore we have predicted large contribution of the light quark/antiquark fragmentation to $D$ mesons at large $x_{F}$, which exeeds the conventional $c / \bar{c} \rightarrow D$ contribution.

The predicted large contributions of $D$ mesons at large $x_{F}$ have important consequences for prompt neutrino flux at large neutrino energies, relevant for the IceCube measurements. We have found that the contribution of the unfavored fragmentation is much more important than the conventional one for large neutrino/antineutrino energies $E_{\nu}>10^{5} \mathrm{GeV}$.

We have calculated in addition the asymmetries for much lower energies $(\sqrt{s}=20-100 \mathrm{GeV})$, relevant for possible measurements in a near future. Much larger asymmetries have been predicted, compared to those measured by the LHCb collaboration [3], even at $y \approx 0$. The asymmetries are associated with an increased production of charm in the $q / \bar{q}$ initiated hadronization. We have quantified this effect by discussing corresponding asymmetries and rapidity distributions. The corresponding measurements at fixed target LHCb, RHIC, and at SPS (NA61-SHINE) would allow to pin down the "new" mechanisms. Especially the SPS experiment could/should observe an enhanced production of $D$ mesons. Even a factor of 5 enhancement is not excluded at present.

We have shown that the calculations within the $k_{T}$-factorization approach as well as the next-to-leading order approach leave considerable room at low energies for the new subleading-fragmentation contribution. Clearly new better quality data are needed at intermediate and low energies to better constrain the subleading fragmentation.

We have also predicted a dependence of the ratio of the charged-to-neutral $D$ meson cross sections as a function of collision energy, meson rapidity or $x_{F}$. We wish to remind in this context that different $K$ factors, relative to pQCD calculations, were found long ago for charged and neutral $D$ meson (see Ref. [41]).

Systematic studies of $D / \bar{D}$ asymmetries or the specific ratios at low energies may be therefore (paradoxically) important to understand the high-energy prompt component of the atmospheric neutrino flux.

\section{ACKNOWLEDGMENTS}

This study was partially supported by the Polish National Science Center Grant No. DEC-2014/15/B/ST2/02528 and by the Center for Innovation and Transfer of Natural 
Sciences and Engineering Knowledge in Rzeszów. A. S. thanks Jolanta Brodzicka and Marcin Chrząszcz for a discussion of the LHCb experiments on D and B meson production. We also thank Victor Goncalves for a discussion on prompt atmospheric flux. The exchange of information on fixed target $\mathrm{LHCb}$ experiments with Michael Winn, Emilie Maurice, and Frederic Fleuret is acknowledged. We also thank Antoni Marcinek and Paweł Staszel for similar information concerining planned NA61SHINE experiment.
[1] M. I. Adamovich et al. (WA82 Collaboration), Phys. Lett. B 305, 402 (1993); G. A. Alves et al. (E769 Collaboration), Phys. Rev. Lett. 77, 2392 (1996); E. M. Aitala et al. (E791 Collaboration), Phys. Lett. B 371, 157 (1996); 411, 230 (1997); M. I. Adamovich et al. (WA92 Collaboration), Nucl. Phys. B495, 3 (1997).

[2] M. I. Adamovich et al. (WA89 Collaboration), Eur. Phys. J. C 8, 593 (1999); 13, 247 (2000).

[3] R. Aaij et al. (LHCb Collaboration), Phys. Lett. B 718, 902 (2013).

[4] P. del Amo Sanchez et al. (BABAR collaboration), Phys. Rev. D 83, 071103 (2011).

[5] B. Ko et al. (Belle collaboration), Phys. Rev. Lett. 109, 021601 (2012).

[6] R. Aaij et al. (LHCb Collaboration), J. High Energy Phys. 10 (2014) 025.

[7] R. Gauld, U. Haisch, B. D. Pecjak, and E. Re, Phys. Rev. D 92, 034007 (2015).

[8] S. J. Brodsky, P. Hoyer, C. Peterson, and N. Sakai, Phys. Lett. 93B, 451 (1980).

[9] T. J. Hobbs, J. T. Londergan, and W. Melnitchouk, Phys. Rev. D 89, 074008 (2014).

[10] P. Jimenez-Delgado, T. J. Hobbs, J. T. Londergan, and W. Melnitchouk, Phys. Rev. Lett. 114, 082002 (2015).

[11] V. A. Bednyakov, S. J. Brodsky, A. V. Lipatov, G. I. Lykasov, M. A. Malyshev, J. Smiesho, and S. Tokar, arXiv:1712.09096.

[12] R. Laha and S. Brodsky, Phys. Rev. D 96, 123002 (2017).

[13] E. R. Cazaroto, V. P. Goncvalves, F. S. Navarro, and M. Nielsen, arXiv:1302.0035; Phys. Lett. B 724, 108 (2013).

[14] F. Halzen and L. Wille, Phys. Rev. D 94, 014014 (2016).

[15] E. Norrbin and T. Sjöstrand, Eur. Phys. J. C 17, 137 (2000).

[16] W. K. Lai, A. K. Leibovich, and A. A. Petrov, Phys. Rev. D 90, 054022 (2014).

[17] R. Rapp and E. V. Shuryak, Phys. Rev. D 67, 074036 (2003).

[18] T. Anticic et al. (NA49 Collaboration), Eur. Phys. J. C 68, 1 (2010).

[19] I. Arsene et al. (BRAHMS collaboration), Phys. Rev. Lett. 98, 252001 (2007).

[20] M. Czech and A. Szczurek, Phys. Rev. C 72, 015202 (2005); J. Phys. G 32, 1253 (2006).

[21] T. Kneesch, B. A. Kniehl, G. Kramer, and I. Schienbein, Nucl. Phys. B799, 34 (2008).
[22] T. Sjstrand, S. Ask, J. R. Christiansen, R. Corke, N. Desai, P. Ilten, S. Mrenna, S. Prestel, C. O. Rasmussen, and P.Z. Skands, Comput. Phys. Commun. 191, 159 (2015).

[23] L. A. Harland-Lang, A. D. Martin, P. Motylinski, and R. S. Thorne, Eur. Phys. J. C 75, 204 (2015).

[24] V. P. Goncalves, R. Maciuła, R. Pasechnik, and A. Szczurek, Phys. Rev. D 96, 094026 (2017).

[25] R. Aaij et al. (LHCb collaboration), Phys. Rev. Lett. 110, 101802 (2013).

[26] R. Aaij et al. (LHCb collaboration), Phys. Rev. Lett. 116, 241801 (2016).

[27] C. Patrignani et al. (Particle Data Group), Chin. Phys. C 40, 100001 (2016).

[28] R. Aaij et al. (LHCb Collaboration), Nucl. Phys. B871, 1 (2013).

[29] V. P. Goncalves, B. Kopeliovich, J. Nemchik, R. Pasechnik, and I. Potashnikova, Phys. Rev. D 96, 014010 (2017).

[30] A. Karpishkov, V. Saleev, and A. Shipilova, Phys. Rev. D 94, 114012 (2016).

[31] R. Maciuła and A. Szczurek, Phys. Rev. D 87, 094022 (2013).

[32] R. Maciuła and A. Szczurek, Phys. Rev. D 94, 114037 (2016).

[33] M. Cacciari, M. Greco, and P. Nason, J. High Energy Phys. 05 (1998) 007; M. Cacciari, S. Frixione, and P. Nason, J. High Energy Phys. 03 (2001) 006.

[34] Y. Ali, P. Staszel, A. Marcinek, J. Brzychczyk, and R. Płaneta, Acta Phys. Pol. B 44, 2019 (2013).

[35] The LHCb collaboration, Report No. LHCb-CONF-2017001.

[36] M. Aguilar-Benitez et al. (LEBC-EHS Collaboration), Z. Phys. C 40, 321 (1988).

[37] R. Ammar et al., Phys. Rev. Lett. 61, 2185 (1988).

[38] A. Bhattacharya, R. Enberg, Y. S. Jeong, C. S. Kim, M. H. Reno, I. Sarcevic, and A. Stasto, J. High Energy Phys. 11 (2016) 167.

[39] R. Maciuła, V. Saleev, A. Shipilova, and A. Szczurek, Phys. Lett. B 758, 458 (2016).

[40] M. G. Aartsen et al. (IceCube collaboration), Astrophys. J. 833, 3 (2016).

[41] P. Braun-Muzinger, D. Miśkowiec, A. Drees, and C. Lourenco, Eur. Phys. J. C 1, 123 (1998). 